\section{Kidney \\ Blood Pressure Research}

\title{
Mortality and Associated Risk Factors in Dialysis Patients with Cardiovascular Disease
}

\author{
Junrong Tong ${ }^{a}$ Manyi Liua,b Hong Lic Zhengmao Luo ${ }^{a} \quad$ Xianyang Zhong ${ }^{a}$ \\ Jun Huang ${ }^{d}$ Riguang Liu ${ }^{d}$ Feng He ${ }^{d}$ Junzhou Fu ${ }^{d}$
}

aDepartment of Nephrology, General Hospital of Guangzhou Military Command of PLA; ${ }^{b}$ Graduate School of Southern Medical University; 'Department of Comprehensive Evaluation, Medical Association of Guangdong Province; dDepartment of Nephrology, Guangzhou First People's Hospital, Guangzhou, China

\section{Key Words}

Cardiovascular disease $\bullet$ Hemodialysis (HD) $\bullet$ Peritoneal dialysis (PD) $\bullet$ Mortality $\bullet$ Risk factors

\begin{abstract}
Background/Aims: Although dialysis patients have a higher risk of morbidity and mortality related to cardiovascular disease (CVD) than the general population, the mortality and associated risk factors in Asian dialysis patients with CVD have not been well examined. Methods: In this prospective cohort study, mortality and risk factors were investigated in 591 dialysis patients who were recruited from two dialysis centers from May 1, 2009 to May 1,2014 . The Cox proportional hazards regression assessed adjusted differences in mortality risk. A multivariate analysis was also performed, comparing the CVD and non-CVD groups. Results: A total of 591 patients were enrolled in this study (mean age, $52.05 \pm 16.46$ years [SD]; $61.8 \%$ men; 20.8\% with CVD), with a median follow-up of 21.9 (maximum, 72) months. The cumulative hazard of mortality was significantly higher in CVD patients (hazard ratio [HR], 1.835; 95\% confidence interval [CI], 1.023-3.293; $P=0.042$ ) than in their non-CVD counterparts after adjusting for various confounders. On multivariate Cox analysis, stroke (HR, 4.574; $95 \%$ CI, 2.149-9.736; $\mathrm{P}<0.001$ ) was an independent predictor of all-cause mortality in the CVD group. In the non-CVD group, diabetes mellitus ( $H R$, 2.974; 95\% $\mathrm{CI}, 1.560-5.668 ; \mathrm{P}=0.001$ ) and elevated high-sensitivity C-reactive lipoprotein (hs-CRP) (HR, 1.017; 95\% CI, 1.005-1.030; $\mathrm{P}=0.005)$ were independent predictors of all-cause mortality. Conclusion: All-cause mortality was significantly higher in the CVD group than in the non-CVD group. Stroke is an independent risk factor for all-cause mortality in dialysis patients with CVD. These findings warrant further studies into preventive and interventional strategies.
\end{abstract}

Junrong Tong, Manyi Liu and Hong Li contributed equally and therefore share first authorship.

Feng $\mathrm{He}$, and

Junzhou Fu
Department of Nephrology, Guangzhou First People's Hospital, $1^{\text {th }}$, Panfu Road, Yuexiu District, 510180 Guangzhou (China) Tel. +862081048188, Fax +862081048112 E-Mail hefengmm@163.com and fujzhou@163.com 


\section{Kidney Blood Pressure Research}

Tong et al.: Mortality and Associated Risk Factors in CVD Dialysis Patients

\section{Introduction}

Chronic kidney disease (CKD) is becoming a common disease in the general population and is associated with an increased risk of mortality [1]. According to the 2013 US Annual Data Report, 615,899 dialysis and transplant patients were receiving treatment for CKD at the end of 2011 [2]. Dialysis patients have a much higher mortality rate than the general population. Registry studies indicated that cardiovascular disease (CVD) is an independent risk factor for and the leading cause of mortality in dialysis patients, accounting for nearly $50 \%$ of deaths in this population [3-7]. The increased incidence of cardiovascular events among dialysis patients is mainly explained by the presence of traditional risk factors, such as prior CVD, elevated C-reactive protein (CRP), left ventricular hypertrophy (LVH), diabetes mellitus, and advanced age [8]. Recent studies examining cardiovascular complications in dialysis patients focused on atherosclerosis, including arterial stiffness and wall thickness changes $[9,10]$. Furthermore, mortality differences by dialysis modality among end-stage renal disease (ESRD) patients with and without CVD were reported, and some studies reported that the mortality risk was not constant over time but increased with follow-up $[11,12]$. The high prevalence of CVD in dialysis patients has drawn great attention from physicians around the world. However, the mortality and risk factors in dialysis patients with CVD has not been fully explored. Therefore, we designed the present prospective, observational cohort study to estimate the mortality rate and risk factors for patients with CVD undergoing hemodialysis (HD) and peritoneal dialysis (PD).

\section{Participants and Methods}

\section{Participants}

From May 1, 2009 to May 1, 2014, 591 patients were recruited from the dialysis centers at General Hospital of Guangzhou Military Command of PLA and Guangzhou First People's Hospital. The follow-up period was from the start of dialysis to dialysis modality change, kidney transplantation, transfer of care away from the two dialysis centers, date of death, withdrew from the study, end of dialysis, or end of the study period, May 1, 2015. Enrollment included patients who were older than 18 years and had received dialysis for more than 3 months. The study protocol was approved by the Clinical Research Ethics Committee of Guangzhou First People's Hospital (Gaungzhou, China) and the Clinical Research Ethics Committee of General Hospital of Guangzhou Military Command of PLA (Guangzhou, China). All patients provided written informed consent before enrollment.

\section{Study protocol}

This study was a prospective, observational cohort study. Baseline data 1 to 3 months after the initiation of dialysis were collected. Demographic information included age, gender, body mass index (BMI), dialysis duration, and comorbidities (diabetes mellitus, hypertension, and stroke). A patient was deemed to have diabetes mellitus if the patient had been diagnosed with type 1 or type 2 diabetes by a clinical doctor. Hypertension was recorded as a comorbidity if the patient was taking antihypertensive medication or had blood pressures greater than $140 / 90 \mathrm{mmHg}$ on two separate occasions. Stroke was defined as an acute disturbance of focal neurological function with symptoms lasting more than 24 hours and considered secondary to intracerebral hemorrhage or ischemia [13]. Clinical data and biochemical parameters included systolic blood pressure (SBP), diastolic blood pressure (DBP), mean arterial pressure (MAP), hemoglobin, serum albumin, hemoglobin A1c (HbA1c), high-sensitivity C-reactive lipoprotein (hs-CRP), phosphorus, calcium, corrected calcium, Ca×P, Kt/V, uric acid, triglycerides, total cholesterol, low-density lipoprotein cholesterol (LDL-C), and high-density lipoprotein cholesterol (HDL-C). The study population was grouped based on the presence or absence of CVD, as well as on the modality of dialysis. CVD was defined by both history of CV event or condition and/or current symptoms according to New York Heart Association (NYHA) and Canadian Cardiovascular Society (CCS) classifications for heart failure and angina, respectively [14]. CV event history was defined as a history of angina pectoris, myocardial infarction, angioplasty, coronary artery disease, peripheral vascular disease, left ventricular hypertrophy, or congestive heart failure [15-17]. All the data were measured in General Hospital of Guangzhou Military Command of PLA and Guangzhou First People's Hospital. The outcome of interest was all-cause mortality. 


\section{Kidney \\ Blood Pressure Research}

Table 1. Baseline characteristics of the study population by dialysis modality and the presence or absence of cardiovascular disease

\begin{tabular}{|c|c|c|c|c|c|c|c|}
\hline \multirow[b]{2}{*}{ Characteristics } & \multirow[b]{2}{*}{ Total (591) } & \multicolumn{3}{|c|}{ HD } & \multicolumn{3}{|c|}{ PD } \\
\hline & & $\mathrm{CVD}(\mathrm{N}=39)$ & Non-CVD $(\mathrm{N}=246)$ & $P$ value & CVD $(\mathrm{N}=84)$ & Non-CVD $(\mathrm{N}=222)$ & $P$ value \\
\hline \multicolumn{8}{|l|}{ Demographics } \\
\hline Age (years) & $52.05 \pm 16.46$ & $70.63 \pm 9.54$ & $55.81 \pm 16.07$ & $<0.001$ & $54.14 \pm 15.64$ & $43.84 \pm 13.53$ & $<0.001$ \\
\hline Male, $n(\%)$ & $365(61.8)$ & $23(59.0)$ & $162(65.9)$ & 0.403 & $51(60.7)$ & $129(58.1)$ & 0.679 \\
\hline Body mass index $\left(\mathrm{kg} / \mathrm{m}^{2}\right)$ & $21.95 \pm 3.35$ & $21.92 \pm 2.16$ & $22.87 \pm 3.70$ & 0.025 & $22.00 \pm 3.14$ & $20.92 \pm 2.89$ & 0.005 \\
\hline Dialysis duration (months) & $26.04 \pm 16.88$ & $16.69 \pm 11.37$ & $22.91 \pm 17.40$ & 0.004 & $31.64 \pm 15.65$ & $28.88 \pm 15.7$ & 0.171 \\
\hline \multicolumn{8}{|l|}{ Comorbidities ( $\mathrm{n} \%$ ) } \\
\hline Diabetes mellitus & $156(26.4)$ & $24(61.5)$ & $70(28.5)$ & $<0.001$ & $29(34.5)$ & $33(14.9)$ & $<0.001$ \\
\hline Hypertension & $405(68.5)$ & $35(89.7)$ & $177(72.0)$ & 0.018 & $57(67.9)$ & $136(61.3)$ & 0.286 \\
\hline Stroke & $41(6.93)$ & $9(23.1)$ & $16(6.50)$ & 0.001 & $11(13.1)$ & $5(2.25)$ & $<0.001$ \\
\hline \multicolumn{8}{|l|}{ Blood pressure } \\
\hline SBP (mmHg) & $141.04 \pm 16.00$ & $142.87 \pm 8.75$ & $145.52 \pm 10.73$ & 0.144 & $138.46 \pm 20.04$ & $136.72 \pm 18.66$ & 0.477 \\
\hline DBP (mmHg) & $82.81 \pm 11.47$ & $78.90 \pm 5.86$ & $80.11 \pm 6.46$ & 0.270 & $83.07 \pm 14.41$ & $86.4 \pm 14.04$ & 0.067 \\
\hline MAP $(\mathrm{mmHg})$ & $102.86 \pm 11.03$ & $101.65 \pm 6.13$ & $103.23 \pm 6.05$ & 0.131 & $101.50 \pm 14.67$ & $103.18 \pm 14.00$ & 0.358 \\
\hline \multicolumn{8}{|l|}{ Laboratory data } \\
\hline Hemoglobin $(\mathrm{g} / \mathrm{L})$ & $95.88 \pm 18.44$ & $92.75 \pm 19.58$ & $92.77 \pm 20.43$ & 0.997 & $97.77 \pm 15.05$ & $99.16 \pm 16.43$ & 0.500 \\
\hline Serum albumin $(\mathrm{g} / \mathrm{L})$ & $36.85 \pm 4.61$ & $34.89 \pm 5.58$ & $36.6 \pm 4.99$ & 0.051 & $36.60 \pm 3.74$ & $37.56 \pm 4.17$ & 0.065 \\
\hline hs-CRP (mg/L) & $5.04(3.11-6.26)$ & $5.03(2.31-7.32)$ & $4.37(1.45-6.42)$ & 0.651 & $4.98(1.74-6.92)$ & $3.07(1.20-6.26)$ & 0.005 \\
\hline HbAlc (\%) & $6.89 \pm 1.02$ & $6.83 \pm 0.98$ & $6.72 \pm 0.64$ & 0.725 & $7.01 \pm 1.03$ & $6.97 \pm 0.99$ & 0.537 \\
\hline Phosphorus (mg/dL) & $5.56 \pm 1.66$ & $5.37 \pm 1.72$ & $6.09 \pm 2.08$ & 0.043 & $5.11 \pm 1.06$ & $5.18 \pm 1.06$ & 0.640 \\
\hline Calcium (mg/dL) & $8.77 \pm 0.99$ & $8.27 \pm 1.26$ & $8.45 \pm 1.12$ & 0.349 & $9.11 \pm 0.68$ & $9.09 \pm 0.69$ & 0.795 \\
\hline Corrected calcium (mmol/L) & $2.19 \pm 0.25$ & $2.07 \pm 0.32$ & $2.11 \pm 0.28$ & 0.356 & $2.28 \pm 0.17$ & $2.27 \pm 0.17$ & 0.795 \\
\hline $\mathrm{Ca} \times \mathrm{P}(\mathrm{mg} / \mathrm{dL})$ & $48.57 \pm 14.76$ & $44.97 \pm 15.76$ & $51.22 \pm 18.58$ & 0.048 & $46.80 \pm 11.27$ & $46.93 \pm 9.75$ & 0.928 \\
\hline Total Kt/V & $1.94 \pm 0.63$ & $1.62 \pm 0.48$ & $1.55 \pm 0.55$ & 0.439 & $2.29 \pm 0.42$ & $2.30 \pm 0.51$ & 0.980 \\
\hline Uric acid (mg/dL) & $7.01 \pm 1.27$ & $6.99 \pm 0.96$ & $7.06 \pm 1.53$ & 0.776 & $6.91 \pm 1.10$ & $7.01 \pm 1.06$ & 0.473 \\
\hline Triglycerides (mmol/L) & $1.67 \pm 0.79$ & $1.82 \pm 0.63$ & $1.70 \pm 0.64$ & 0.280 & $1.77 \pm 0.91$ & $1.57 \pm 0.89$ & 0.096 \\
\hline Total cholesterol $(\mathrm{mmol} / \mathrm{L})$ & $4.65 \pm 1.13$ & $4.30 \pm 0.90$ & $4.26 \pm 1.05$ & 0.820 & $5.18 \pm 1.08$ & $4.96 \pm 1.09$ & 0.119 \\
\hline $\mathrm{HDL}-\mathrm{C}(\mathrm{mmol} / \mathrm{L})$ & $2.59 \pm 0.75$ & $2.24 \pm 0.62$ & $2.29 \pm 0.62$ & 0.664 & $2.91 \pm 0.78$ & $2.86 \pm 0.75$ & 0.564 \\
\hline LDL-C (mmol/L) & $1.70 \pm 0.70$ & $2.23 \pm 0.52$ & $2.20 \pm 0.62$ & 0.826 & $1.20 \pm 0.34$ & $1.24 \pm 0.40$ & 0.340 \\
\hline
\end{tabular}

\section{Statistical analysis}

Data were expressed as mean \pm standard deviation for continuous normally distributed variables, frequencies (percentages) for categorical variables, and median (interquartile range) for continuous nonnormally distributed variables. Characteristic differences between two groups were tested using Student's t-test for approximately normally distributed continuous variables, chi-squared test for categorical variables, and the non-parametric Mann-Whitney U test for skewed continuous variables. Life tables were used to calculated cumulative survival rates during follow-up. Univariate Cox regression was used to evaluate prognostic risks for all-cause mortality among baseline parameters in the whole population and in the CVD and non-CVD groups. Then, the significant variables from the univariate model were placed in multivariate Cox regression to further analyze possible risk factors (entry: $\mathrm{P} \leq 0.05$; removal: $\mathrm{P}>0.1$ ). Survival rates were calculated using the Kaplan-Meier method, and the difference between survival rates was analyzed using the log-rank test. A Cox proportional hazards model was constructed for the CVD and non-CVD groups. Multivariate models were constructed assessing first the group, then sequentially adding 1) the demographic characteristics; 2) comorbidities; 3) SBP, DBP, and MAP; and 4) laboratory values. All statistical test values were two-tailed tests, and $\mathrm{P}<0.05$ was considered statistically significant. Data were analyzed using SPSS, version 20.0 for Windows (SPSS, Chicago, IL, USA).

\section{Results}

Baseline characteristics of the study cohort

Table 1 contains the demographic and clinical data at baseline, categorized by the presence or absence of CVD history. The study cohort consisted of 591 patients (mean age, $52.05 \pm 16.46$ years [SD]; $61.8 \%$ men; $20.8 \%$ with CVD). Of those, 285 underwent HD, and the remainder underwent PD. The median follow-up period lasted 21.9 (maximum, 72) months. Reasons for exclusion were loss to follow-up $(n=6)$, renal transplantation $(n=25)$, withdrawal from the study ( $n=8)$, and transfer to non-participating dialysis centers $(n=9)$. In HD patients, patients with CVD were older and had higher rates of diabetes mellitus, hypertension, and stroke, but lower BMIs, dialysis duration, phosphorus, and Ca×P than patients without CVD. 


\section{Kidney \\ Blood Pressure Research}

Kidney Blood Press Res 2016;41:479-487

DOI: $10.1159 / 000443449$

Published online: July 20, 2016

C 2016 The Author(s). Published by S. Karger AG, Basel www.karger.com/kbr

Fig. 1. The hazard ratios with $95 \%$ confidence interval for the prediction of all-cause mortality in dialysis patients with CVD. (Model 1: Univariate analysis of risk in CVD patients. Model 2: adjusted for age, sex, BMI. Model 3: adjusted for model 2 covariates and comorbidities. Model 4: adjusted for model 3 covariates and SBP, DBP, MAP. Model 5: adjusted for model 4 covariates and hemoglobin, serum albumin, hs-CRP, total cholesterol, triglycerides, phosphorus, calcium, Ca×P, HbAlc, Kt/V, uric acid, LDL-C, HDL-C.).

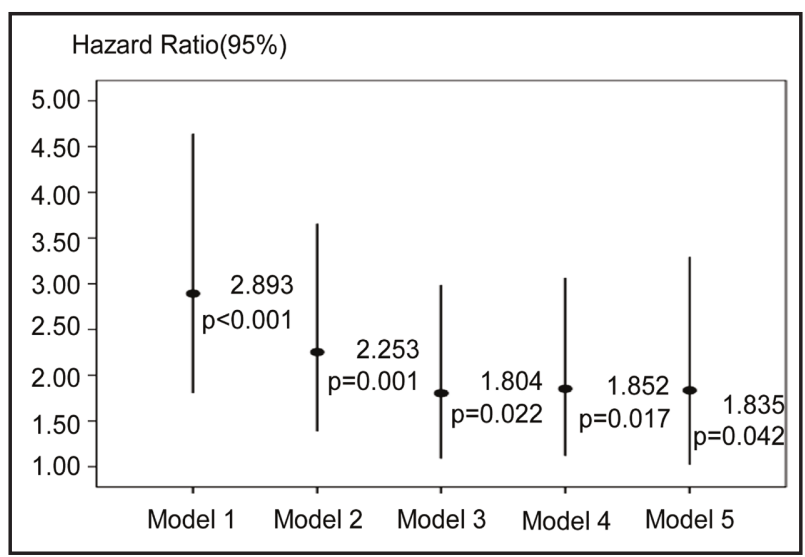

In PD patients, patients with CVD had higher rates of diabetes mellitus and stroke, as well as higher BMIs and hs-CRP values.

\section{Mortality analysis}

During the study period, 70 fatalities occurred, 31 of which were in the CVD group. Multivariate analysis revealed significant association between CVD and all-cause mortality (Figure 1). In the maximally adjusted model, which included demographics, comorbidities, and clinical data, the adjusted HR was 1.835 (95\% CI, 1.023-3.293; P=0.042). In addition, the risk of death was higher in the CVD group than in the non-CVD group with HRs of 2.893 (95\% CI, 1.804-4.693; $\mathrm{P}<0.001$ ), 4.072 (95\% CI, 2.036-8.142; $\mathrm{P}<0.001$ ), and 3.803 (95\% CI, 1.8457.843; $\mathrm{P}<0.001$ ) in all patients, patients who underwent HD, and patients who underwent PD, respectively (Figure 2). The mortality rates in the CVD group were 18\%, 43\%, and 53\% at 1,3 , and 5 years, respectively, compared with $8 \%, 13 \%$, and $24 \%$, respectively, in the nonCVD group. Moreover, in CVD patients, the risk of death was significantly higher in the HD group than in the PD group (HR, 1.569; 95\% CI, 1.032-2.392; $\mathrm{P}<0.001)$ (Figure 3). In nonCVD patients, the risk of death was also higher in the HD group than in the PD group (HR, 1.218; 95\% CI, 1.109-1.348; $\mathrm{P}<0.001$ ).

\section{Risk factors for all-cause mortality}

Clinical data and laboratory variables that were significantly different in Table 1 , as well as the importance of clinical concern were included in the univariate Cox regression analysis. Table 2 lists the significant risk factors for all-cause mortality by adjusting for covariates that were statistically significant in the univariate Cox regression analysis. In the total cohort, stroke (HR, 3.614; 95\% CI, 2.043-6.393; P<0.001), CVD (HR, 2.580; 95\% CI, 1.4664.540; $\mathrm{P}=0.001)$ and dialysis modality (HR, 2.169; 95\% CI, 1.230-3.831; $\mathrm{P}=0.007$ ) were independent predictors of all-cause mortality after adjusting for age, diabetes mellitus, DBP, serum albumin, hs-CRP, and HbAlc. The predictors of all-cause mortality in patients with and without CVD are summarized in Table 3. In the CVD group, stroke (HR, 4.574; 95\% CI, 2.149-9.736; $\mathrm{P}<0.001$ ) was an independent predictor of all-cause mortality after adjusting for age, corrected calcium, calcium, HbAlc and dialysis modality. Additionally, in the nonCVD group, diabetes mellitus (HR, 2.974; 95\% CI, 1.560-5.668; P=0.001) and elevated hsCRP (HR, 1.017; 95\% CI, 1.005-1.030; $\mathrm{P}=0.005$ ) were independent predictors of all-cause mortality after adjusting for age, stroke, hemoglobin, HbAlc, total cholesterol, HDL-C and dialysis modality.

\section{Discussion}

The results of this study indicated that all-cause mortality was significantly higher in the CVD group than in the non-CVD group among dialysis patients. Importantly, we found that the mortality risk factors were stroke, and CVD and dialysis modality in the overall dialysis 


\section{Kidney Blood Pressure Research}
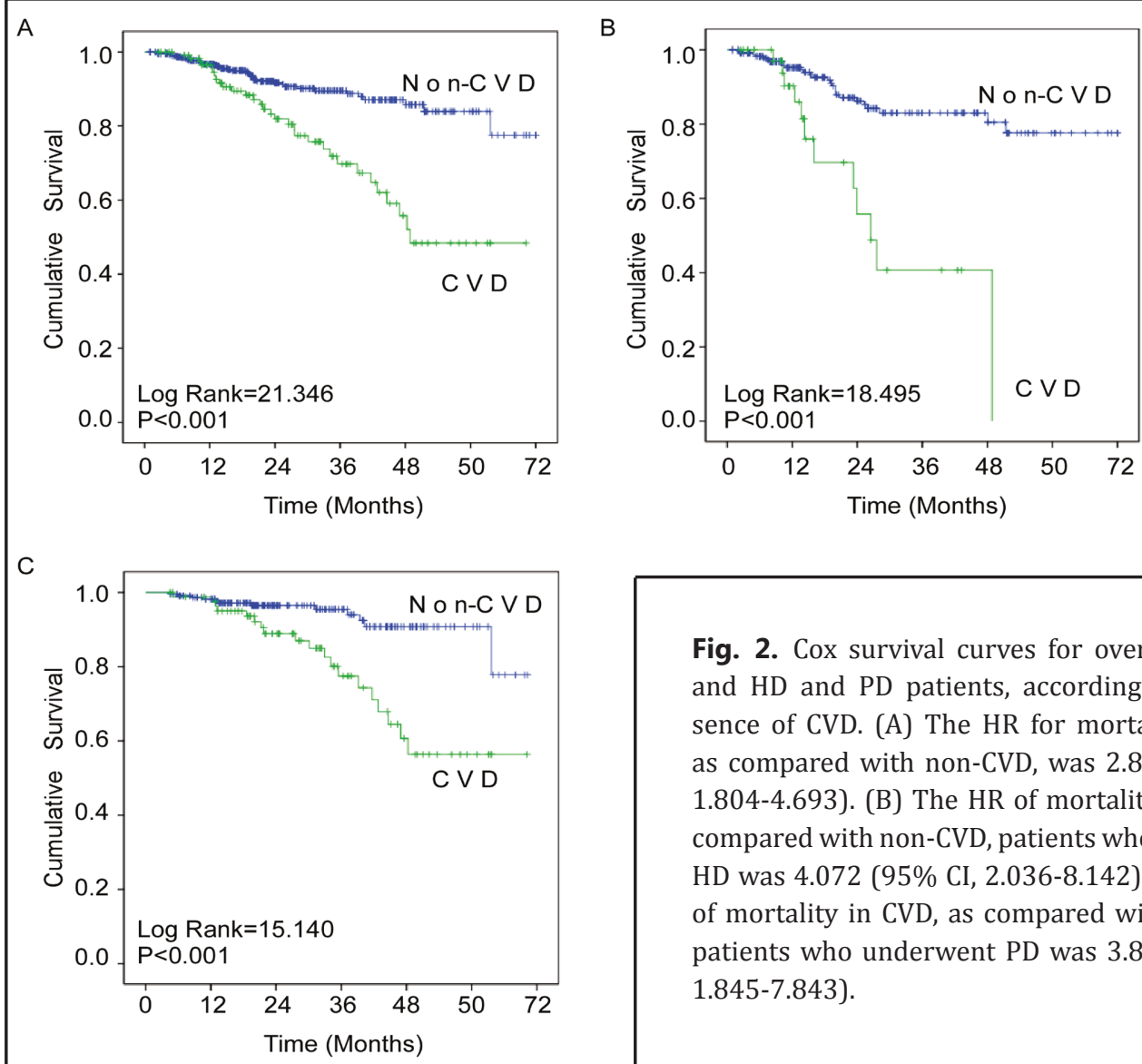

Fig. 2. Cox survival curves for overall patients, and HD and PD patients, according to the presence of CVD. (A) The HR for mortality in CVD, as compared with non-CVD, was 2.893 (95\% CI, 1.804-4.693). (B) The HR of mortality in CVD, as compared with non-CVD, patients who underwent HD was 4.072 (95\% CI, 2.036-8.142). (C) The HR of mortality in CVD, as compared with non-CVD, patients who underwent PD was 3.803 (95\% CI, 1.845-7.843).

A

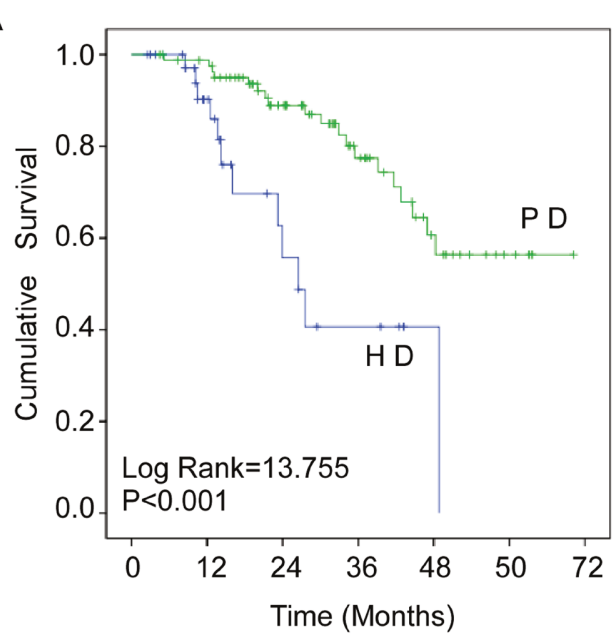

B

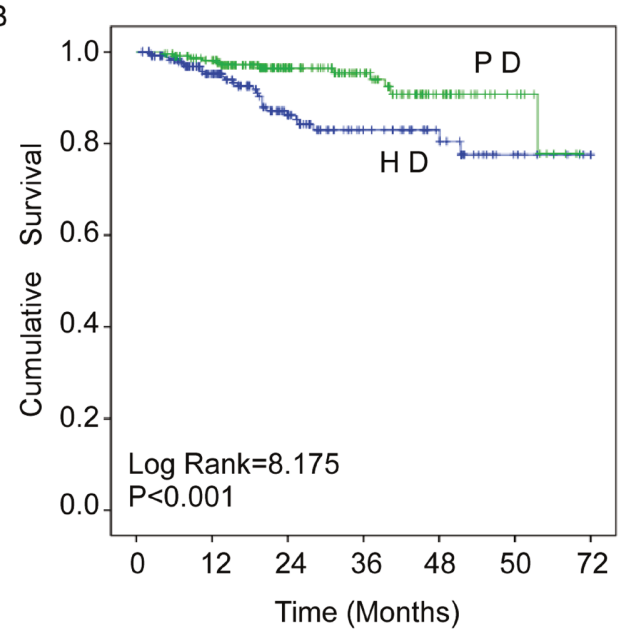

Fig. 3. Cox survival curves for CVD and non-CVD patients according to dialysis modality. (A) The HR of mortality in HD, as compared with PD, patients in the CVD group was 1.569 (95\% CI, 1.032-2.392). (B) The HR of mortality in HD, as compared with PD, patients in the non-CVD group was 1.218 (95\% CI, 1.109-1.348).

population. Stroke was an independent prognostic predictor for mortality in CVD patients, whereas diabetes mellitus and higher hs-CRP were independent risk factors for mortality in non-CVD patients. 


\section{Kidney Blood Pressure Research}

Table 2. Predictor variables and multivariate model of all-cause mortality in dialysis patients

\begin{tabular}{lccccc}
\hline \multirow{2}{*}{ PatiCharacteristics } & \multicolumn{2}{c}{ Univariate Analysis } & & \multicolumn{2}{c}{ Multivariate Analysis } \\
\cline { 2 - 3 } \cline { 5 - 6 } Age (years) & HR $(95 \% \mathrm{CI})$ & $P$ value & & HR $(95 \% \mathrm{CI})$ & $P$ value \\
Diabetes mellitus & $2.040(1.025-1.057)$ & $<0.001$ & & - & - \\
Stroke & $5.642(3.319-3.416)$ & 0.002 & & - & - \\
CVD & $2.893(1.804-4.639)$ & $<0.001$ & & $2.580(1.466-4.540)$ & 0.001 \\
DBP (mmHg) & $0.976(0.957-0.995)$ & 0.015 & & - & - \\
Serum albumin (g/L) & $0.951(0.912-0.991)$ & 0.016 & & - & - \\
hs-CRP (mg/L) & $1.019(1.008-1.031)$ & 0.001 & & - & - \\
HbAlc (\%) & $1.062(1.028-1.097)$ & $<0.001$ & & - & - \\
Dialysis modality* & $1.912(1.191-3.067)$ & 0.007 & & $2.169(1.230-3.831)$ & 0.007 \\
\hline
\end{tabular}

*Dialysis modality HD versus PD (reference); Abbreviations: HR, hazard ratio; CI, confidence interval; CVD, cardiovascular disease; DBP, Diastolic blood pressure; hs-CRP, high-sensitivity C-reactive protein; HbAlc, hemoglobin A1c

Table 3. Predictor variables and multivariate model for all-cause mortality in dialysis patients with and without cardiovascular disease

\begin{tabular}{|c|c|c|c|c|}
\hline Characteristics & Unadjusted HR (95\% CI) & $P$ value & Adjusted HR (95\% CI) & $P$ value \\
\hline \multicolumn{5}{|c|}{ CVD (123) } \\
\hline Age (years) & $1.053(1.020-1.088)$ & 0.002 & - & - \\
\hline Stroke & $4.591(2.241-9.405)$ & $<0.001$ & $4.574(2.149-9.736)$ & $<0.001$ \\
\hline Corrected calcium (mmol) & $0.243(0.087-0.681)$ & 0.007 & _ & - \\
\hline Calcium (mg/dL) & $0.702(0.542-0.908)$ & 0.007 & _- & - \\
\hline HbAlc (\%) & $1.078(1.046-1.110)$ & $<0.001$ & - & - \\
\hline Dialysis modality* & $1.569(1.032-2.392)$ & $<0.001$ & & \\
\hline \multicolumn{5}{|c|}{ Non-CVD (468) } \\
\hline Age (years) & $1.027(1.006-1.048)$ & 0.010 & - & - \\
\hline Diabetes mellitus & $3.223(1.716-6.053)$ & $<0.001$ & $2.974(1.560-5.668)$ & 0.001 \\
\hline Stroke & $4.036(1.575-10.345)$ & 0.004 & - & - \\
\hline Hemoglobin $(\mathrm{g} / \mathrm{L})$ & $0.984(0.968-1.000)$ & 0.046 & - & - \\
\hline hs-CRP (mg/L) & $1.021(1.009-1.033)$ & $<0.001$ & $1.017(1.005-1.030)$ & 0.005 \\
\hline HbAlc $(\%)$ & $1.074(1.025-1.126)$ & 0.003 & - & - \\
\hline Total cholesterol (mmol/L) & $1.033(1.013-1.053)$ & 0.001 & _- & _- \\
\hline HDL-C (mmol/L) & $0.599(0.406-0.884)$ & 0.010 & _- & _ \\
\hline Dialysis modality* & $1.218(1.109-1.348)$ & $<0.001$ & & \\
\hline \multicolumn{5}{|c|}{$\begin{array}{l}\text { *Dialysis modality HD versus PD (reference); Abbreviations: HR, hazard ratio; CI, confidence interval; } \\
\text { CVD, cardiovascular disease; hs-CRP, high-sensitivity C-reactive protein; HbAlc, hemoglobin A1c; } \\
\text { HDL-C, high-density lipoprotein cholesterol }\end{array}$} \\
\hline
\end{tabular}

It is a widely held opinion that CVD is associated with worse survival rates in dialysis patients. Other studies have shown that CVD-related mortality in dialysis patients is 10 to 20 times higher than in the general population [7]. Chung et al. [3]reported that the mortality risk for DM dialysis patients with CVD is 3.3 times higher than that for non-DM dialysis patients without CVD. In the Modification of Diet in Renal Disease Study of nondiabetic chronic renal insufficiency patients, $25 \%$ of first hospitalizations, about 3 per 100 patientyears, were due to CVD [7]. The outcomes of these studies were consistent with our findings. In our study, all-cause mortality occurred at a rate of 54.7 deaths/1,000 patient-years for all patients, 112.4 deaths/1,000 patient-years in the CVD group, and 38.85 deaths/1,000 patient-years in the non-CVD group.

The high mortality in dialysis patients with CVD may be explained by the high prevalence of diabetes mellitus in dialysis patients, which may impact vascular access maturity. Arterial stiffness and vascular calcification deteriorate during dialysis, which may lead to CVD [18]. Diabetes and ESRD synergistically increase the risk of cardiovascular events [19]. Another possible explanation for the high mortality in CVD dialysis patients is fluid overload, which is associated with hypertension and LVH in this population. These pathophysiologic changes 


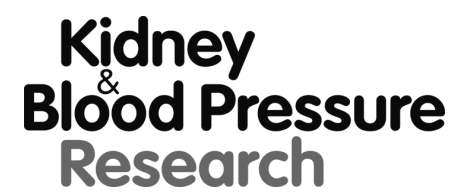

Kidney Blood Press Res 2016;41:479-487
\begin{tabular}{l|l}
\hline DOI: $10.1159 / 000443449$ & (c) 2016 The Author(s). Published by S. Karger AG, Basel \\
Published online: July 20, 2016 & www.kargercom/kbr
\end{tabular}

are multifactorial. The progressive loss of renal function and dialysis itself may contribute to the progression of CVD [20].

Different risks factors were found to be associated with mortality when the study population was divided into patients with and without CVD. In CVD patients, stroke was an independent predictor of all-cause mortality, while diabetes mellitus and hs-CRP were independent predictors of all-cause mortality in the non-CVD group. Stroke is the third leading cause of death in the United States and a leading cause of serious disability [21]. Wang et al. [22] showed that patients undergoing dialysis are at an elevated risk of stroke. In addition, older age, male sex, diabetes, and hypertension, which are risk factors for CVD, are independent risk factors for both ischemic and hemorrhagic strokes [23, 24]. Our findings were consistent with that study, which included 80,000 dialysis patients. Dialysis patients have a high prevalence of CVD, which is a risk factor for incident stroke [23]. Chan et al. [25] reported that CVD is a common comorbidity in patients with ESRD, and warfarin use in dialysis patients may confer additional risk for stroke in HD patients. Previous reports suggested all-cause mortality in dialysis patient with CVD is closely associated with traditional risk factors, such as lipid abnormality, hyperhomocysteinemia, abdominal obesity, chronic inflammation, hypoalbuminemia, oxidative stress and advanced glycosylation end-products formation, electrolyte imbalances, hemodynamic overload, and cardiovascular history [4-6, $8,16,26,27]$. In addition, poor sleep quality was found to be a risk factor for CVD in a recent study [17]. Therefore, although these factors were not found to be significantly associated with all-cause mortality among CVD patients in our study, they should be noted by clinicians when managing dialysis patients.

Only a few large-scale studies have measured the effect of dialysis modality on mortality in Asian populations. PD is considered the better choice for ESRD patients because of its positive effect on hemodynamic stability and volume regulation [11]. Likewise, our study found decreased survival rates in CVD dialysis patients treated with HD as compared to the rates of those treated with PD, which is consistent with the findings of prior studies [28,29]. In our study, among CVD dialysis patients, the HR of all-cause death in HD as compared with PD was 1.569; among non-CVD dialysis patients, the HR of all-cause death in HD patients as compared to PD patients was 1.218. However, our findings may be inconsistent with some previous studies, which found that the mortality among dialysis patients with CVD was not constant over time but increased with follow-up [11, 12, 30]. Johnson et al. [31] reported that the risk of death from CVD was significantly increased in PD patients compared with HD patients after the first year of treatment. Therefore, large-scale investigation is needed to evaluate the potential explanations for these findings.

This study had some limitations. First, patients were not randomly assigned to PD or HD. This could have led to results that were biased based on the criteria used to determine whether a patient would receive HD or PD. Second, the recruited patients were mainly from South China, which may affect the generalization of our findings to other regions. Third, due to the limitation of the number of patients, not all the cardiovascular classification were shown in our study, which deserve us to further investigation by expanding sample size. Finally, other dialysis-related factors, such as medication use, severity of comorbidities, nutrition, parameters of quality of life, and the cause of the deaths that could have affected the results were not evaluated because of incomplete information.

\section{Conclusion}

Our study explored the mortality and associated risk factors in Asian dialysis patients with CVD. We found that the cumulative hazard of mortality was significantly higher in CVD patients than in their non-CVD counterparts after adjusting for various confounders. Furthermore, stroke was confirmed as a significant risk factor for mortality in the CVD group. These findings may warrant additional monitoring of dialysis patients with CVD, especially those with stroke. Further studies are necessary to devise preventive and interventional strategies for dialysis patients with CVD. 


\section{Kidney \\ Blood Pressure Research} \begin{tabular}{l|l}
\hline Kidney Blood Press Res 2016;41:479-487 \\
\hline DOI: $10.1159 / 000443449$ & (c) 2016 The Author(s). Published by S. Karger AG, Basel \\
Published online: July 20, 2016 & www.karger.com/kbr
\end{tabular}

\section{Disclosure Statement}

The authors declare that they have no competing interests.

\section{Acknowledgments}

We thank the trial nurses in the two dialysis centers. We express our gratitude to all patients who participated in the study. This work was supported by grants from the National Natural Science Foundation of China (Grant No. 81270895; 81360137) and the Guangdong Natural Science Foundation (Grant No. 2014A030310060; 2014A020212253; 2016A030313490).

\section{References}

1 Zhang QL, Rothenbacher D: Prevalence of chronic kidney disease in population-based studies: Systematic review. BMC Public Health 2008;8:117.

2 Collins AJ, Foley RN, Chavers B, Gilbertson D, Herzog C, Ishani A, Johansen K, Kasiske BL, Kutner N, Liu J, St PW, Guo H, Hu Y, Kats A, Li S, Li S, Maloney J, Roberts T, Skeans M, Snyder J, Solid C, Thompson B, Weinhandl E, Xiong H, Yusuf A, Zaun D, Arko C, Chen SC, Daniels F, Ebben J, Frazier E, Johnson R, Sheets D, Wang X, Forrest B, Berrini D, Constantini E, Everson S, Eggers P, Agodoa L: US Renal Data System 2013 Annual Data Report. Am J Kidney Dis 2014;63:A7.

3 Chung SH, Han DC, Noh H, Jeon JS, Kwon SH, Lindholm B, Lee HB: Risk factors for mortality in diabetic peritoneal dialysis patients. Nephrol Dial Transplant 2010;25:3742-3748.

4 Prichard S: Major and minor risk factors for cardiovascular disease in continuous ambulatory peritoneal dialysis patients. Perit Dial Int 1999;19:S133-S137.

5 Locatelli F, Pozzoni P, Tentori F, Del VL: Epidemiology of cardiovascular risk in patients with chronic kidney disease. Nephrol Dial Transplant 2003;18:i2-i9.

6 Foley RN, Murray AM, Li S, Herzog CA, McBean AM, Eggers PW, Collins AJ: Chronic kidney disease and the risk for cardiovascular disease, renal replacement, and death in the United States Medicare population, 1998 to 1999. J Am Soc Nephrol 2005;16:489-495.

7 Foley RN, Parfrey PS, Sarnak MJ: Clinical epidemiology of cardiovascular disease in chronic renal disease. Am J Kidney Dis 1998;32:S112-S119.

8 Kessler M, Zannad F, Lehert P, Grunfeld JP, Thuilliez C, Leizorovicz A, Lechat P: Predictors of cardiovascular events in patients with end-stage renal disease: An analysis from the Fosinopril in dialysis study. Nephrol Dial Transplant 2007;22:3573-3579.

9 Maekawa K, Shoji T, Emoto M, Okuno S, Yamakawa T, Ishimura E, Inaba M, Nishizawa Y: Influence of atherosclerosis on the relationship between anaemia and mortality risk in haemodialysis patients. Nephrol Dial Transplant 2008;23:2329-2336.

10 Zapolski T, Wysokinski A, Ksiazek A, Jaroszynski A: Aortic stiffness and left atrial volume index in patients on continuous ambulatory peritoneal dialysis: Role of endothelial dysfunction. Int J Cardiol 2013;162:253256.

11 Stack AG, Molony DA, Rahman NS, Dosekun A, Murthy B: Impact of dialysis modality on survival of new ESRD patients with congestive heart failure in the United States. Kidney Int 2003;64:1071-1079.

12 Ganesh SK, Hulbert-Shearon T, Port FK, Eagle K, Stack AG: Mortality differences by dialysis modality among incident ESRD patients with and without coronary artery disease. J Am Soc Nephrol 2003;14:415-424.

13 Stroke-1989. Recommendations on stroke prevention, diagnosis, and therapy. Report of the WHO Task Force on Stroke and other Cerebrovascular Disorders. Stroke 1989;20:1407-1431.

14 Levin A, Djurdjev O, Barrett B, Burgess E, Carlisle E, Ethier J, Jindal K, Mendelssohn D, Tobe S, Singer J, Thompson C: Cardiovascular disease in patients with chronic kidney disease: Getting to the heart of the matter. Am J Kidney Dis 2001;38:1398-1407. 


\section{Kidney \\ Blood Pressure Research}

Kidney Blood Press Res 2016;41:479-487

DOI: $10.1159 / 000443449$

Published online: July 20, 2016

(C) 2016 The Author(s). Published by S. Karger AG, Base www.karger.com/kbr

15 Davies SJ, Russell L, Bryan J, Phillips L, Russell GI: Comorbidity, urea kinetics, and appetite in continuous ambulatory peritoneal dialysis patients: Their interrelationship and prediction of survival. Am J Kidney Dis 1995;26:353-361.

16 Mou S, Wang Q, Fang W, Lin A, Shi B, Cao L, Zhou W, Qian J, Ni Z: Change in cardiovascular disease status in peritoneal dialysis patients: A 5-year single-center experience. Ren Fail 2012;34:28-34.

17 Zhang J, Wang C, Gong W, Peng H, Tang Y, Li CC, Zhao W, Ye Z, Lou T: Association between sleep quality and cardiovascular damage in pre-dialysis patients with chronic kidney disease. BMC Nephrol 2014;15:131.

18 Panday VB, Tong Z, Ng PL, Lee EJ, Lau T, Teo B, Chua H: Dialysis modality and 2-year outcomes in patients with ischemic cardiomyopathy and end-stage renal disease. Int J Cardiol 2014;176:1097-1099.

19 Chang YT, Wu JL, Hsu CC, Wang JD, Sung JM: Diabetes and end-stage renal disease synergistically contribute to increased incidence of cardiovascular events: A nationwide follow-up study during 1998-2009. Diabetes Care 2014;37:277-285.

20 Broers NJH, Cuijpers ACM, van der Sande FM, Leunissen KML, Kooman JP: The first year on haemodialysis: A critical transition. Clin Kidney J 2015;8:271-277.

21 Covic A, Schiller A, Mardare NG, Petrica L, Petrica M, Mihaescu A, Posta N: The impact of acute kidney injury on short-term survival in an Eastern European population with stroke. Nephrol Dial Transplant 2008;23:2228-2234.

22 Wang HH, Hung SY, Sung JM, Hung KY, Wang JD: Risk of stroke in long-term dialysis patients compared with the general population. Am J Kidney Dis 2014;63:604-611.

23 Drew DA, Sarnak MJ: Ischemic and hemorrhagic stroke: High incidence in hemodialysis and peritoneal dialysis patients. Am J Kidney Dis 2014;63:547-548.

24 Nakayama M, Metoki H, Terawaki H, Ohkubo T, Kikuya M, Sato T, Nakayama K, Asayama K, Inoue R, Hashimoto J, Totsune K, Hoshi H, Ito S, Imai Y: Kidney dysfunction as a risk factor for first symptomatic stroke events in a general Japanese population--the Ohasama study. Nephrol Dial Transpl 2007;22:19101915.

25 Chan KE, Lazarus JM, Thadhani R, Hakim RM: Warfarin use associates with increased risk for stroke in hemodialysis patients with atrial fibrillation. J Am Soc Nephrol 2009;20:2223-2233.

26 Gosmanova EO, Le NA: Cardiovascular complications in CKD patients: Role of oxidative stress. Cardiol Res Pract 2011;2011:156326.

27 Carrero JJ, de Jager DJ, Verduijn M, Ravani P, De Meester J, Heaf JG, Finne P, Hoitsma AJ, Pascual J, Jarraya F, Reisaeter AV, Collart F, Dekker FW, Jager KJ: Cardiovascular and noncardiovascular mortality among men and women starting dialysis. Clin J Am Soc Nephrol 2011;6:1722-1730.

28 Murphy SW, Foley RN, Barrett BJ, Kent GM, Morgan J, Barre P, Campbell P, Fine A, Goldstein MB, Handa SP, Jindal KK, Levin A, Mandin H, Muirhead N, Richardson RM, Parfrey PS: Comparative mortality of hemodialysis and peritoneal dialysis in Canada. Kidney Int 2000;57:1720-1726.

29 Korevaar JC, Feith GW, Dekker FW, van Manen JG, Boeschoten EW, Bossuyt PM, Krediet RT: Effect of starting with hemodialysis compared with peritoneal dialysis in patients new on dialysis treatment: A randomized controlled trial. Kidney Int 2003;64:2222-2228.

30 Glynn LG, Reddan D, Newell J, Hinde J, Buckley B, Murphy AW: Chronic kidney disease and mortality and morbidity among patients with established cardiovascular disease: A West of Ireland community-based cohort study. Nephrol Dial Transplant 2007;22:2586-2594.

31 Johnson DW, Dent H, Hawley CM, McDonald SP, Rosman JB, Brown FG, Bannister K, Wiggins KJ: Association of dialysis modality and cardiovascular mortality in incident dialysis patients. Clin J Am Soc Nephrol 2009;4:1620-1628. 